\title{
Effect of running coupling on photon emission from quark gluon plasma
}

\author{
Mahatsab Mandal and Pradip Roy \\ Saha Institute of Nuclear Physics, 1/AF Bidhannagar Kolkata - 700064, India \\ Sukanya Mitra and Sourav Sarkar \\ Variable Energy Cyclotron Centre, 1/AF Bidhannagar Kolkata - 700064, India
}

\begin{abstract}
We discuss the role of running coupling on the thermal photon yield from quark gluon plasma. It is shown that the photon production rate from the partonic phase is considerably enhanced when running coupling is considered with respect to a fixed value. However, we show by explicit evaluation that although this difference survives the space-time evolution the experimental data cannot distinguish between the two once the hard contribution, which is an essential component of photon production mechanism, is added.
\end{abstract}

Detection of quark gluon plasma (QGP) in heavy ion collisions has received significant attention in recent years. Among possible signals, electromagnetic probes are one of the most promising tools to characterize the initial state of the collisions [1]. Owing to their weak coupling with the constituents of the system they tend to escape almost unscattered. In fact, photons (dileptons as well) can be used to determine the initial temperature, or equivalently the equilibration time. By comparing the initial temperature with the transition temperature from lattice QCD, one can infer whether QGP is produced.

Photons are produced at various stages from (i) initial hard scattering of partons, (ii) scattering of charged particles in the thermal medium (QGP and hadronic matter) and (iii) from $\pi^{0}$ and $\eta^{0}$ decays. If this decay contribution is subtracted from the total photon yield what is left is the direct (excess) photons. The thermal photon rate due to Compton and annihilation processes in a quark gluon plasma has been calculated by several authors over the last two decades [2 6]. In all these calculations the strong coupling, $\alpha_{s}$ is treated as constant or function of temperature $T$. However, in case of relativistic heavy ion collisions, apart from temperature there is also the momentum scale $k$. One has to take into account the case when $k \sim T$ and treat $\alpha_{s}$ to be function of both $k$ and $T$ [7]. By incorporating this fact it is shown that the energy loss is a factor of $2-6$ more than the case when $\alpha_{s}$ is constant 7, 8]. The energy loss calculation using running coupling and reduced screening mass in [8], explains single electron $R_{A A}$ quite well. It is the purpose of this brief report to treat the strong coupling as running and apply it to the case of thermal photon production from QGP.

The lowest order processes for photon emission from QGP are the Compton scattering $(q(\bar{q}) g \rightarrow q(\bar{q}) \gamma)$ and annihilation $(q \bar{q} \rightarrow g \gamma)$ process. The total cross-section diverges in the limit $t$ or $u \rightarrow 0$. These singularities have to be shielded by thermal effects in order to obtain infrared safe calculations. It has been argued in Ref. 9] that the intermediate quark acquires a thermal mass in the medium, whereas the hard thermal loop (HTL) approach of Ref. [3] shows that very soft modes are suppressed in a medium providing a natural cut-off $k_{c} \sim g T$. We assume that the singularities can be shielded by the introduction of thermal masses for the participating partons. The differential cross-sections for Compton and annihilation processes are given by [10],

$$
\begin{aligned}
& \frac{d \sigma(q g \rightarrow q \gamma)}{d \hat{t}}=\frac{1}{6}\left(\frac{e_{q}}{e}\right)^{2} \frac{8 \pi \alpha_{s} \alpha_{e}}{\left(\hat{s}-m^{2}\right)^{2}}\left(\frac{m^{2}}{\hat{s}-m^{2}}+\frac{m^{2}}{\hat{u}-m^{2}}\right)^{2} \\
& +\left(\frac{m^{2}}{\hat{s}-m^{2}}+\frac{m^{2}}{\hat{u}-m^{2}}\right)-\frac{1}{4}\left(\frac{\hat{s}-m^{2}}{\hat{u}-m^{2}}+\frac{\hat{u}-m^{2}}{\hat{s}-m^{2}}\right)
\end{aligned}
$$

and

$$
\begin{aligned}
& \frac{d \sigma(q \bar{q} \rightarrow g \gamma)}{d \hat{t}}=-\frac{4}{9}\left(\frac{e_{q}}{e}\right)^{2} \frac{8 \pi \alpha_{s} \alpha_{e}}{\hat{s}\left(\hat{s}-4 m^{2}\right)}\left(\frac{m^{2}}{\hat{t}-m^{2}}+\frac{m^{2}}{\hat{u}-m^{2}}\right)^{2} \\
& +\left(\frac{m^{2}}{\hat{t}-m^{2}}+\frac{m^{2}}{\hat{u}-m^{2}}\right)-\frac{1}{4}\left(\frac{\hat{t}-m^{2}}{\hat{u}-m^{2}}+\frac{\hat{u}-m^{2}}{\hat{t}-m^{2}}\right)
\end{aligned}
$$

where $m$ is the in-medium thermal quark mass, $m^{2} \equiv$ $m_{t h}{ }^{2}=2 \pi \alpha_{s} T^{2} / 3, \alpha_{e}$ and $\alpha_{s}$ are the electromagnetic fine-structure and the strong coupling constants respectively. The static photon rate in $1+2 \rightarrow 3+\gamma$ can be written as [1]

$$
\begin{aligned}
\frac{d N}{d^{4} x d^{2} p_{T} d y}= & \frac{\mathcal{N}_{i}}{(2 \pi)^{7} E} \int d \hat{s} d \hat{t}\left|\mathcal{M}_{i}\right|^{2} \times \int d E_{1} d E_{2} \\
& \frac{f_{1}\left(E_{1}\right) f_{2}\left(E_{2}\right)\left(1+f_{3}\left(E_{3}\right)\right)}{\sqrt{a E_{2}{ }^{2}+2 b E_{2}+c}}
\end{aligned}
$$

where

$$
\begin{aligned}
& a=-\left(\hat{s}+\hat{t}-m_{2}{ }^{2}-m_{3}{ }^{2}\right)^{2} \\
& b=E_{1}\left(\hat{s}+\hat{t}-m_{2}{ }^{2}-m_{3}{ }^{2}\right)\left(m_{2}{ }^{2}-\hat{t}\right) \\
& +E\left[\left(\hat{s}+\hat{t}-m_{2}{ }^{2}-m_{3}{ }^{2}\right)\right. \\
& \left.\times\left(\hat{s}-m_{1}{ }^{2}-m_{2}{ }^{2}\right)-2 m_{1}{ }^{2}\left(m_{2}{ }^{2}-\hat{t}\right)\right] \\
& c=-E_{1}^{2}\left(m_{2}^{2}-\hat{t}\right)^{2} \\
& \text { - } 2 E_{1} E\left[2 m_{2}^{2}\left(\hat{s}+\hat{t}-m_{2}^{2}-m_{3}^{2}\right)\right. \\
& \text { - } \left.\left(m_{2}^{2}-\hat{t}\right)\left(\hat{s}-m_{1}^{2}-m_{2}^{2}\right)\right] \\
& \text { - } E^{2}\left[\left(\hat{s}-m_{1}{ }^{2}-m_{2}^{2}\right)^{2}-4 m_{1}{ }^{2} m_{2}^{2}\right] \\
& -\left(\hat{s}+\hat{t}-m_{2}{ }^{2}-m_{3}^{2}\right)\left(m_{2}^{2}-\hat{t}\right) \\
& \times\left(\hat{s}-m_{1}{ }^{2}-m_{2}{ }^{2}\right)+m_{2}{ }^{2}\left(\hat{s}+\hat{t}-m_{2}{ }^{2}-m_{3}{ }^{2}\right)^{2} \\
& +m_{1}^{2}\left(m_{1}^{2}-\hat{t}\right)^{2}
\end{aligned}
$$




$$
\begin{aligned}
& E_{1, \text { min }}=\frac{\hat{s}+\hat{t}-m_{2}^{2}-m_{3}^{2}}{4 E}+\frac{E m_{1}{ }^{2}}{\hat{s}+\hat{t}-m_{2}{ }^{2}-m_{3}^{2}} \\
& E_{2, \text { min }}=\frac{E m_{2}^{2}}{m_{2}^{2}-\hat{t}}+\frac{m_{2}^{2}-\hat{t}}{4 E}, E_{2, \text { max }}=-\frac{b}{a}+\frac{\sqrt{b^{2}-a c}}{a} .
\end{aligned}
$$

$\mathcal{M}_{i}$ represents the amplitude for Compton or annihilation process. The overall degeneracy factor $\mathcal{N}_{i}=320 / 3$ and 20 for Compton and annihilation processes respectively involving $u$ and $d$ quarks.

As mentioned earlier, the infra-red cut-off is fixed by plasma effects, where only the medium part is considered, completely neglecting the vacuum contribution leading to ambiguity in the calculation of cross-section at finite temperature QCD. If the latter part is taken into account the strong coupling should be running. Thus for any consistent calculation one has to take this fact into consideration. We have in that case $\alpha_{s}=\alpha_{s}(k, T)$ where $k=\sqrt{|t|}$.

Photons from thermal hadronic matter also make up an essential component of the total photon yield from heavy ion collisions. These are emitted in reactions between charged hadrons and in the radiative decays of unstable hadrons 1, 11, 12. In this work, we have used the amplitudes of photon producing reactions involving the $\pi, \rho, \omega, \eta, K$ and $K^{*}$ mesons obtained in [13].

The hard photon contribution can be calculated by perturbative QCD (pQCD). In order to calculate photon production from reactions of the type $h_{A} h_{B} \rightarrow \gamma X$ (where $h_{A}, h_{B}$ refer to hadrons), we assume that the energy is such that the partonic degrees of freedom become relevant and they behave incoherently. The crosssection for this process can then be written in terms of elementary parton-parton cross-section multiplied by the partonic flux which depends on the parton distribution functions [14]. The energy scale for this to happen i.e. the factorization scale is denoted by $Q^{2}$, the square of the momentum transfer of the reaction. Starting with two body scattering at the partonic level the differential cross-section for the reaction of above type can be written as 15.

$$
\begin{aligned}
\frac{d \sigma_{\gamma, \mathrm{hard}}}{d^{2} p_{T} d y} & =K \sum_{a b c} \int_{x_{a}^{\min }}^{1} d x_{a} G_{a / h_{A}}\left(x_{a}, Q^{2}\right) G_{b / h_{B}}\left(x_{b}, Q^{2}\right) \\
& \times \frac{2}{\pi} \frac{x_{a} x_{b}}{2 x_{a}-x_{T} e^{y}} \frac{d \sigma}{d \hat{t}}(a b \rightarrow \gamma c)
\end{aligned}
$$

where, $x_{T}=2 p_{T} / \sqrt{s}$ and the factor $K$ is introduced to take into account the higher order effects. A few comments about the $K$ factor is in order here. The crosssection in the above expression is calculated perturbatively to leading order (LO) in the strong coupling. In cases where the next-to-leading (NLO) order terms are comparable to the LO terms the $K$ factor defined as $\mathrm{NLO} / \mathrm{LO}$ is introduced in the $\mathrm{LO}$ computations to bring in the essence of the NLO terms. It has been shown in [16] that $K$ depends on the choice of the momentum scale, the parton distribution functions and the shadow-

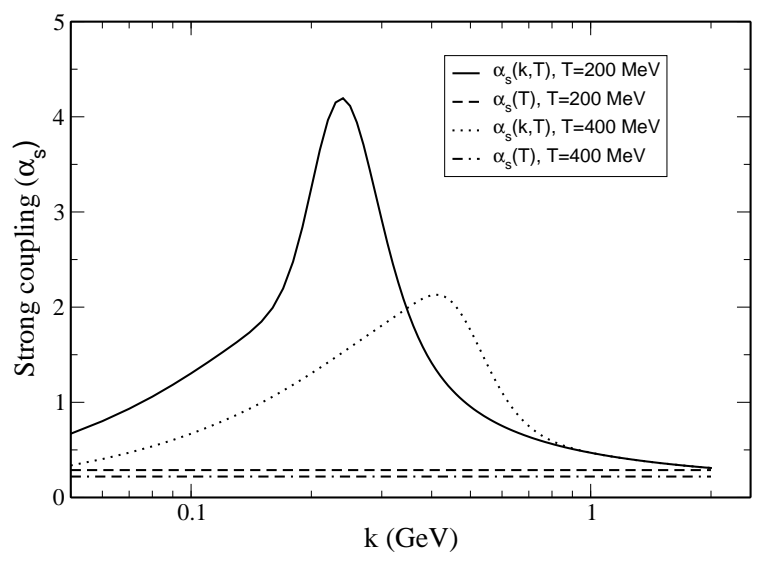

FIG. 1: Strong coupling as a function of momentum scale at two different temperatures [7].

ing effect and its value lies between $2-3$. In the present calculation we take $K \sim 2.5$.

We also include photons from fragmentation process. This is accomplished by introducing the fragmentation function, $D_{\gamma / c}\left(z, Q^{2}\right)$, which when multiplied by $d z$ gives the probability for obtaining a photon from parton $c, z$ being the fractional momentum carried by the photon. Once the photon production cross-section is obtained from hadron-hadron collision we can now determine the direct photon production rates due to hard scattering between partons from nucleus-nucleus collisions at relativistic energies. To do this we must note that the experimental data are given for a particular centrality. In order to take this into account we introduce the centrality parameter which depends on the maximum impact parameter $b_{m}$. The photon yield from hard collisions is then calculated from the expression:

$$
\frac{d N_{\mathrm{AB}}}{d^{2} p_{T} d y}\left(b_{m}\right)=\mathcal{R}\left(b_{m}\right)\left[\frac{d \sigma_{\gamma, \text { hard }}}{d^{2} p_{T} d y}+\frac{d \sigma_{\gamma, \text { frag }}}{d^{2} p_{T} d y}\right]
$$

where $\mathcal{R}\left(b_{m}\right) \equiv\left\langle A B T_{\mathrm{AB}}\right\rangle=\frac{\int_{0}^{b_{m}} d^{2} \mathbf{b} A B T_{\mathrm{AB}}(b)}{\int_{0}^{b_{m}} d \mathbf{b}\left(1-\left[1-T_{\mathrm{AB}}(b) \sigma_{N N}^{i n}\right]^{A B}\right)}$ and $T_{\mathrm{AB}}(\mathbf{b})=\int d^{2} \mathbf{s} T_{\mathrm{A}}(\mathbf{s}) T_{\mathrm{B}}(\mathbf{b}-\mathbf{s})$ is the nuclear overlap function. For $0-10 \%$ centrality we obtain $\mathcal{R} \sim$ $21.7 \mathrm{mb}^{-1}$. Before going to the numerical evaluation of the static photon rate we plot the running coupling in Fig. (1) where the parametrization for $\alpha_{s}(k, T)$ is taken from Ref. [7]. It is seen that the value of the coupling is largest when $k \sim T$. For $k>>T$ it agrees well with the temperature dependent coupling. These features of $\alpha_{s}$ have important consequence on the photon production rate as we shall see below.

The static photon rate is obtained from Eq.(3) using the running coupling. For $T=200 \mathrm{MeV}$ the rates are shown in Fig. (2). The photon emission rate is enhanced by a factor of 1.7 - 6 compared to the case when the momentum dependence of the strong coupling is neglected. 


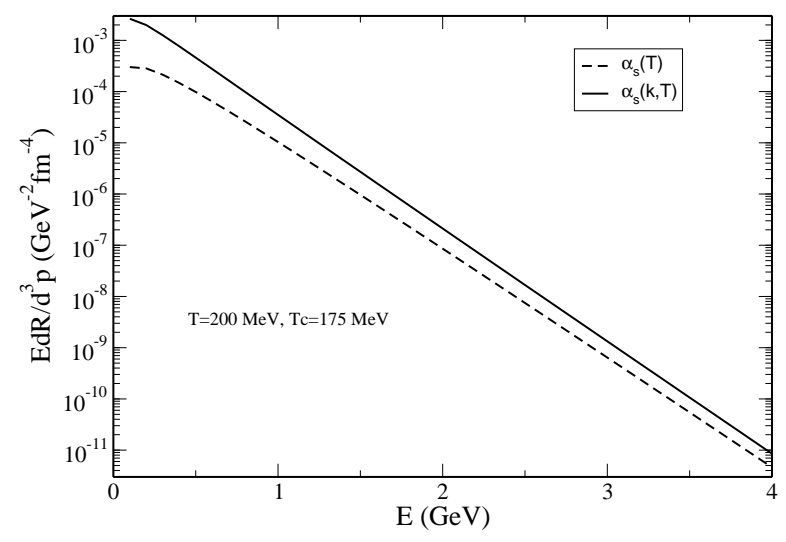

FIG. 2: Static photon rate with and without the running coupling.

Photons are produced at all stages of the collision and so it is necessary to integrate the emission rates over the space-time volume from creation to freeze-out. We assume that quark gluon plasma having a temperature $T_{i}$ is produced at an initial time $\tau_{i}$. Hydrodynamic expansion and cooling follows up to a temperature $T_{c}$ where QGP crosses over to a hadronic gas. Subsequent cooling leads to freeze-out of the fluid element into observable hadrons. In the present work the fireball is taken to undergo an azimuthally symmetric transverse expansion along with a boost invariant longitudinal expansion. The local temperature of the fluid element and the associated flow velocity as a function of the radial coordinate and proper time is obtained by solving the the energy momentum conservation equation $\partial_{\mu} T^{\mu \nu}=0$ where $T^{\mu \nu}=(\epsilon+P) u^{\mu} u^{\nu}+g^{\mu \nu} P$ is the energy momentum tensor for ideal fluid. This set of equations are closed with the Equation Of State (EoS); typically a functional relation between the pressure $P$ and the energy density $\epsilon$. It is a crucial input which essentially controls the profile of expansion of the fireball. To minimize model dependencies we take the EoS from the lattice calculations of the Wuppertal-Budapest Collaboration [17.

The initial temperature is related to the experimentally measured hadron multiplicity through entropy conservation [18] as $T_{i}^{3}\left(b_{m}\right) \tau_{i}=\frac{2 \pi^{4}}{45 \zeta(3) \pi R_{A}^{2} 4 a_{k}}\left\langle\frac{d N}{d y}\left(b_{m}\right)\right\rangle$ where $\left\langle d N / d y\left(b_{m}\right)\right\rangle$ is the hadron (predominantly pions) multiplicity for a given centrality class with maximum impact parameter $b_{m}, R_{A}$ is the transverse dimension of the system and $a_{k}$ is the degeneracy of the system created. The hadron multiplicity resulting from $A u+A u$ collisions is related to that from $p p$ collision at a given impact parameter and collision energy through the relation $\left\langle\frac{d N}{d y}\left(b_{m}\right)\right\rangle=\left[(1-x)\left\langle N_{\text {part }}\left(b_{m}\right)\right\rangle / 2+x\left\langle N_{\text {coll }}\left(b_{m}\right)\right\rangle\right] \frac{d N_{p p}}{d y}$ where $x$ is the fraction of hard collisions. $\left\langle N_{\text {part }}\right\rangle$ is the average number of participants and $\left\langle N_{\text {coll }}\right\rangle$ is the average number of collisions evaluated by using Glauber model. $d N_{p p}^{c h} / d y=2.5-0.25 \ln s+0.023 \ln ^{2} s$ is the multiplicity of the produced hadrons in $p p$ collisions at centre
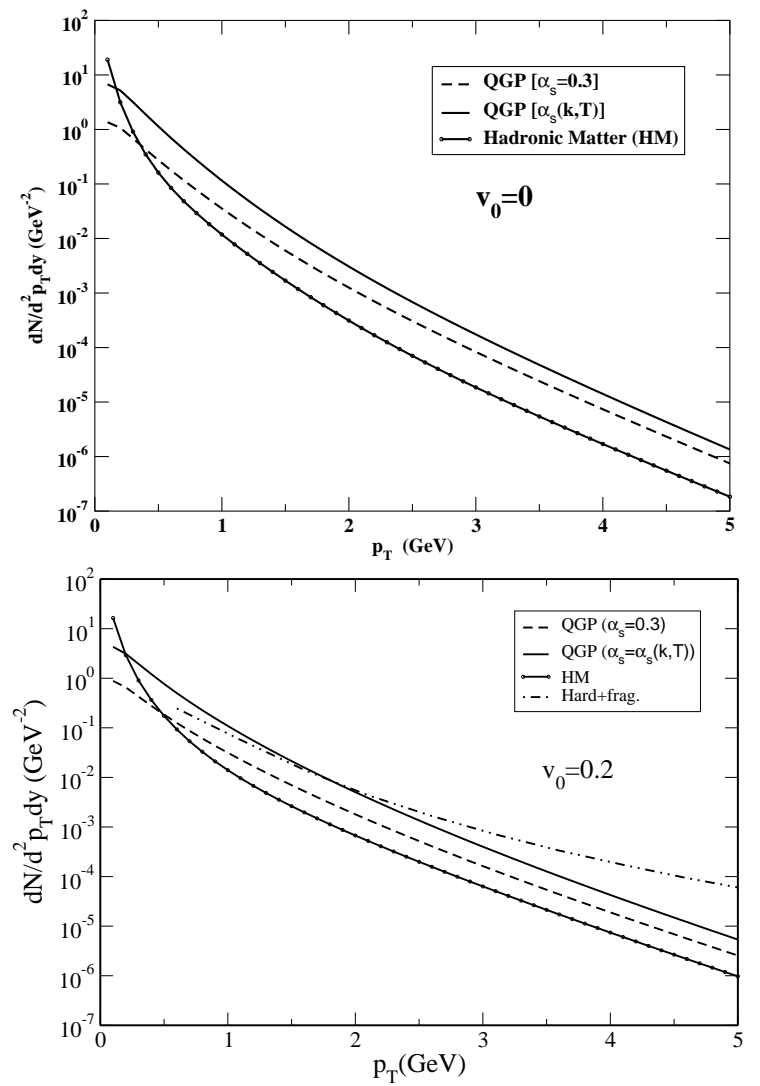

FIG. 3: Thermal photon $p_{T}$ distributions for $T_{i}=400 \mathrm{MeV}$ and $\tau_{i}=0.2 \mathrm{fm} / \mathrm{c}$ at central rapidity. Upper panel corresponds to $v_{0}=0$. Individual contribution from hard and fragmentation photons has been shown in the lower panel for $v_{0}=0.2$.

of mass energy, $\sqrt{s}[19]$. We have assumed that $20 \%$ hard (i.e. $x=0.20$ ) and $80 \%$ soft collisions are responsible for initial entropy production. Considering 0 - $10 \%$ centrality we get $T_{i}=400 \mathrm{MeV}$ for $\tau_{i}=0.2$ $\mathrm{fm} / \mathrm{c}$. One also requires the initial energy density and radial velocity profiles which are taken respectively as [20] $\epsilon\left(\tau_{i}, r\right)=\frac{\epsilon_{0}}{1+e^{\left(r-R_{A}\right) / \delta}}$ and $v\left(\tau_{i}, r\right)=v_{0}\left[1-\frac{1}{1+e^{\left(r-R_{A}\right) / \delta}}\right]$, where $\delta(\sim 0.5 \mathrm{fm})$ is a parameter, known as the surface thickness. As discussed in [20], this choice of the initial fluid velocity profile is motivated by the fact that for a physical system the initial fluid velocity is zero inside the matter which approaches a value $v_{0}$ which is of the order of a typical particle transverse velocity in the diffuse region outside the matter distribution.

The other inputs are the transition temperature $T_{c}$ which is taken as $175 \mathrm{MeV}$ as obtained from lattice QCD 21, 22] and the freeze-out temperature, $T_{f}$ which is taken to be $120 \mathrm{MeV}$. We now plot the thermal photon yield from both QGP and hot hadronic matter in Fig.(3) for $v_{0}=0$ and 0.2 in the upper and lower panels. At very low $p_{T} \sim 0.5 \mathrm{GeV}$, the contribution from hadronic matter dominates. Beyond that the QGP contribution 


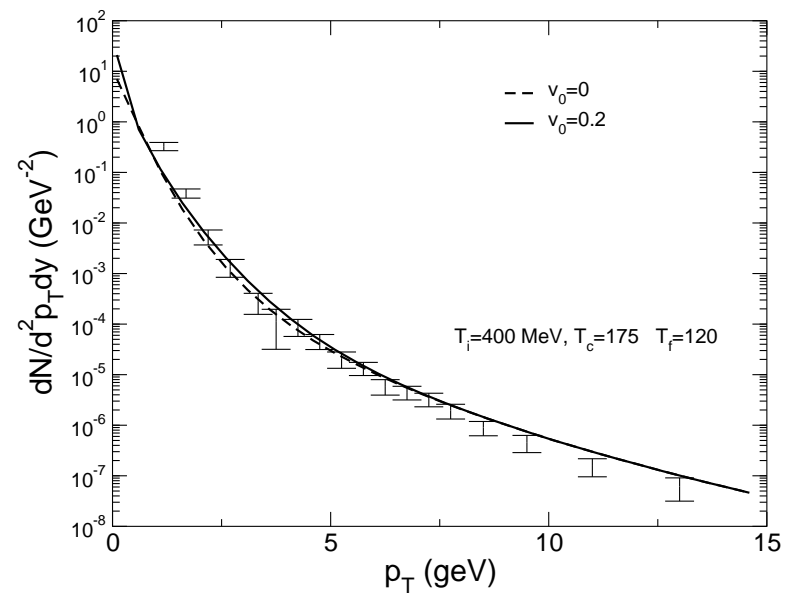

FIG. 4: Photon $p_{t}$ distributions for $T_{i}=400 \mathrm{MeV}$ and $\tau_{i}=$ $0.2 \mathrm{fm} / \mathrm{c}$ at central rapidity. The data (for $|y| \leq 0.35$ ) are taken from Ref. [23].

starts to take over. Interestingly, the effect of running coupling on the thermal photon production from QGP does survive the space time evolution and continues to be discernible in this $p_{T}$ domain. The effect of non-zero initial velocity $\left(v_{0}\right)$ is also visible in the upward shift of the spectra at higher $p_{T}$ in the lower panel compared to the upper. Moreover, the relative separation between the QGP contributions with and without the running coupling appears to be independent of the space-time evolution scenarios corresponding to $v_{0}=0$ and 0.2 . To assess their relative importance in comparison to the thermal yield we also show in the lower panel the contribution from hard QCD photons and photons produced from jet fragmentation. As shown by the dash-dotted line this contribution clearly dominates the photon yield beyond about $2 \mathrm{GeV}$. Below that the hard and fragmentation contributions are almost similar to QGP contribution.
We now compare the total yield with the direct photon data from $\mathrm{Au}+\mathrm{Au}$ collisions at $\mathrm{RHIC}$ obtained by PHENIX [23] in fig. (44). It is observed that the data is best reproduced by assuming a small initial velocity of the order of 0.2 (solid line) compared to $v_{0}=0$ (dashed line). However, the curves for $\alpha_{s}=\alpha_{s}(T)$ and $\alpha_{s}=\alpha_{s}(k, T)$ have merged with each other implying that the observed difference seen in the thermal photon contribution for the two cases has been washed away once the hard and fragmentation contributions are added. Such a result can be understood once we realize that the contribution at a given value of the transverse momentum, especially up to $2-3 \mathrm{GeV} / \mathrm{c}$ is a superposition of contributions from QGP at temperatures from $T_{i}$ to $T_{c}$, hadronic matter from $T_{c}$ to $T_{f}$ as well as from hard scatterings. Although the QGP contribution clearly dominates for $p_{T}>0.5 \mathrm{GeV}$ observation of momentum-dependence of the strong coupling in the transverse momentum spectra of single photons does not appear to be feasible as it is overshadowed by the contributions coming from initial hard collisions.

To summarize, we have calculated the static photon rate from QGP due to Compton and annihilation processes using the temperature and momentum dependent strong coupling. The rate is then contrasted with the case where $\alpha_{s}$ depends only on the temperature of the system. It is found that the static photon rate enhances significantly if the running coupling is used. We then perform a space-time evolution using relativistic hydrodynamics with initial conditions for $\mathrm{Au}+\mathrm{Au}$ collisions at $200 \mathrm{GeV} / \mathrm{n}$ at RHIC. The significant difference in the yields does survive the space time evolution and can be observed in the thermal photon spectra. However, due to the large contributions coming from the initial hard collisions and jet fragmentation the single photon data from PHENIX cannot distinguish between the scenarios with and without momentum dependence of the running coupling.
[1] J. Alam, S. Sarkar, P. Roy, T. Hatsuda, and B. Sinha, Ann. Phys. 286159 (2000).

[2] J. Kapusta, P. Lichard, and D. Seibert, Phys. Rev. D44 2774 (1991).

[3] R. D. Pisarski and E. Braaten, Nucl. Phys. B337, 569 (1990); ibid 339, 310 (1990).

[4] P. Auranche, F. Gelis, H. Zaraket, and R. Kobes, Phys. Rev. D58 (1998) 085003

[5] P. Auranche, F. Gelis, and H. Zaraket, Phys. Rev. D61 (2000) 116001; ibid D62 (2000) 096012.

[6] P. Arnold, G. D. Moore, and L. G. Yaffe, J. High Energy Phys. 11 (2001) 057; ibid 12 (2001) 009;06 (2002) 030; T. renk, Phys. Rev. C67 (2003) 064901.

[7] J. Braun and H-J. Pirner, Phys. Rev. D75, 054031 (2007).

[8] P. B. Gossiaux and A. Aichelin, Phys. Rev. C78, 014904 (2008).

[9] K. Kajantie and P. V. Ruuskanen Phys. Lett. B121, 352
(1983).

[10] C. Y. Wong and H. Wang, Phys. Rev. C58, 376 (1998).

[11] S. Sarkar, J. Alam, P. Roy, A. K. Dutt-Mazumder, B. Dutta-Roy and B. Sinha, Nucl. Phys. A 634206 (1998).

[12] P. Roy, S. Sarkar, J. Alam and B. Sinha Nucl. Phys. A 653277 (1999).

[13] S. Turbide, R. Rapp and C. Gale, Phys. ReV. C 69, 014903(2004).

[14] J. Pumplin, D. R. Stump, J.Huston, H. L. Lai, P. Nadolsky, W. K. Tung, J. High Energy Phys. 0120207 (2002).

[15] J. F. Owens, Rev. Mod. Phys. 59465 (1987).

[16] K. J. Eskola and K. Tuominen, Phys. Rev. D 63114006 (2001).

[17] S. Borsanyi et al., JHEP 1011 (2010) 077

[18] R. C. Hwa R C and K. Kajantie, Phys. Rev.D32, 1109 (1985).

[19] D. Khazreev and M. Nardi, Phys. Lett. B507, 121 (2001)

[20] H. von Gersdorff, M. Kataja, L. McLerran and P. V. 
Ruuskanen, Phys. Rev.D34 794; ibid. D34 (1986).

[21] Katz S 2006 Nucl. Phys. A 774159.

[22] Cheng M et al. 2006 Phys. Rev. D 74054507.

[23] S. S. Adler et al., Phys. Rev. Lett. 94, 232301 (2005); S.
S. Adler et al., Phys. Rev. Lett. 98, 012002 (2007); A. Adare et al., PHENIX Collaboration, arXiv:0804.4168. 